Meta

Journal des traducteurs

Translators' Journal

\title{
La traduction proligère : allocution d'ouverture
}

\section{André Clas}

Volume 35, numéro 1, mars 1990

Actes du colloque international " La traduction proligère "

URI : https://id.erudit.org/iderudit/002281ar

DOI : https://doi.org/10.7202/002281ar

Aller au sommaire du numéro

Éditeur(s)

Les Presses de l'Université de Montréal

ISSN

0026-0452 (imprimé)

1492-1421 (numérique)

Découvrir la revue

Citer ce document

Clas, A. (1990). La traduction proligère : allocution d'ouverture. Meta, 35(1),

10-12. https://doi.org/10.7202/002281ar d'utilisation que vous pouvez consulter en ligne.

https://apropos.erudit.org/fr/usagers/politique-dutilisation/ 


\title{
LA TRADUCTION PROLIGÈRE: ALLOCUTION D'OUVERTURE
}

ANDRÉ CLAS

Université de Montréal, Montréal, Canada

\begin{abstract}
Dévorante passion, mais nullement gratuite, du même en d'autres mots, qui sature d'immenses bibliothèques de traductions. Infinie sollicitude pour le dire babélique. Les plus fous le prennent même comme sa propre fin. À l'affût de la plus parfaite adéquation de messages homosèmes en deux systèmes hétérogènes, cette passion n'est qu'une autre face de l'amour des langues.
\end{abstract}

(Claude Hagège 1985 : 383-384)

Permettez-moi de vous souhaiter la plus cordiale bienvenue et de vous dire que je suis très heureux de vous voir tous ici. Je voudrais vous dire encore que je suis très fier d'ouvrir ce colloque devant autant d'intervenants illustres, tous spécialistes et passionnés du domaine qui nous réunit. J'aimerais vous rappeler qu'il s'agit d'un colloque, c'està-dire, selon le dictionnaire, d'un «débat entre plusieurs personnes sur des questions de doctrine», et c'est bien de cela qu'il s'agit, d'un débat. C'est donc affirmer que les questions, les interventions et les interrogations sont sollicitées. Le dictionnaire ajoute une remarque intéressante à l'entrée colloque: «Débat organisé avec moins de participants que le congrès.» On ne précise pas ce «moins». Je me permettrai donc, pour avoir regardé autour de moi, de dire que ce colloque est un congrès, et tant mieux !

Peut-être me faut-il fournir quelques indications sur le titre choisi : La Traduction proligère. Le qualificatif a pu surprendre certains, mais pour ma part, je le trouve tout à fait bien choisi. Vous me répliquerez que cela n'a rien d'étonnant puisque j'en suis le responsable. Certes! Mais la valeur de cet adjectif des sciences naturelles semble tout à fait s'appliquer à l'activité traduisante: porter descendance selon l'étymologie (proles: «descendance» et gerere: «porter»), «qui porte un germe»! C'est ce sens pour la traduction que je veux retenir, ce sens qui indique une fécondation, le développement, la création de nouvelles idées, l'intégration, le changement, l'enrichissement par l'Autre, mais aussi à cause de l'Autre.

Ce titre me semblait encore tout particulièrement bien choisi pour marquer le $35^{\mathrm{e}}$ anniversaire de notre revue. En effet, en octobre 1955 paraît une petite brochure intitulée Journal des traducteurs / Translators'Journal publiée par l'Association canadienne des traducteurs diplômés. Ces quelques feuilles sont devenues, avec l'âge, la revue que vous connaissez. C'est presque une pause publicitaire! Mais je me permettrai de rappeler que la revue a participé de façon volontaire et intense à l'évolution des idées, des techniques et des choses. Je pense pouvoir dire que la revue était présente dans le changement, qu'elle a joué son rôle et qu'elle mérite qu'on souligne son $35^{\mathrm{e}}$ anniversaire avec éclat.

Ce titre de Traduction proligère est encore bien choisi, car il souligne l'importance de la traduction dans l'évolution des civilisations. Je pense plus particulièrement aux origines de la traduction si l'on se fie à la Bible, où, dans Genèse II, Yahweh a confondu les deux langues. C'est, à mon sens, un immense bienfait et non une malédiction, car Yahweh a donné une nouvelle leçon à l'humanité en lui disant de séparer le divin de ce qui est humain. Yahweh a voulu signifier que la diversité des langues est un bienfait pour 
l'humanité ; elle nourrit la pluralité des civilisations et permet, par comparaison, la pensée neuve, la découverte.

On voit d'ailleurs, puisque nous en sommes à la Bible, l'importance fondamentale de la traduction. Notre civilisation judéo-chrétienne s'appuie sur les traductions bibliques. Rappelons, en passant, que la traduction de la Bible par Luther est le plus important monument de la langue allemande.

La traduction est aussi à l'origine de la Réforme. La traduction a dé-sacré la langue religieuse en tant que valeur en soi pour montrer que le mélange divin, social, politique et linguistique ne tient plus. Certains cherchent encore de nos jours à maintenir ou à rétablir ce mélange!

La traduction, c'est encore la Renaissance, le ressourcement aux origines avec de nouvelles vues sur l'opération traduisante, c'est aussi la Querelle des Anciens et des Modernes. «Les conduites complexes chez l' homme sont un legs de la culture. L'acquis culturel sédimente lentement, mais il n'est pas génétiquement transmissible, il implique l'effort soutenu, il passe par la retransmission culturelle, la langue, le bouche à oreille, le savoir-faire et ouï-dire ... l'utilisation des codes, les écritures, les alphabets», écrit Pierre Chaunu (1989: 54). La traduction c'est donc encore la littérature et les influences des uns sur les autres. C'est Adélard de Bath, qui traduit de l'arabe en latin, c'est La Fontaine et les fables d'Ésope, c'est Grimm et Blanche-Neige, c'est Perrault et le Petit Chaperon Rouge, la liste est longue, $n$ 'insistons pas. «(...) la traduction constitue d'emblée un paradigme de la communication interculturelle, outre qu'elle est un point de passage obligé permettant de passer par-dessus les verrous linguistiques», écrit Jean-René Ladmiral (1989: 30), résumant ainsi parfaitement ce que nous voulons dire.

La traduction proligère, c'est en plus les problèmes théoriques et les cheminements sur la traduction, les méthodologies. Les difficultés et les problèmes de la traduction, ni ses réussites et ses joies, ne datent d'aujourd'hui, mais sont déjà évoqués par Cicéron, saint Jérôme, du Bellay, Étienne Dolet, par exemple. De nos jours, ce sont encore ces mêmes questions qui intéressent, mais les noms ont changé et les spécialistes sont devant nous!

On a souvent cité le développement inouï des communications depuis la fin de la Deuxième Guerre mondiale comme facteur d'augmentation du volume de la traduction. C'est vrai, on traduit énormément et on traduit plus rapidement. C'est un fait que l'augmentation de la consommation de tout a provoqué une traduction de tout. C'est tout à fait normal et c'est bien ainsí. Le nombre des consommateurs a augmenté, mais le fait demeure. On a toujours traduit, moins dans le passé, peut-être parce qu'il y avait moins de producteurs, moins de production et moins de consommateurs! La traduction est tout simplement une nécessité pour tous et qui a toujours une fonction proligère. C'est vrai, on vient de le voir pour la littérature. Mais c'est aussi vrai pour les sciences et les technologies, pour la politique. La recherche, la découverte, l'invention, le procédé, le gadget est proligère. La vie est proligère! «La diversité des langues nourrit celle des civilisations et par là contribue à façonner les traits uniques qui définissent l'homme», écrit Claude Hagège (1987: 13). Elles nourrissent les civilisations parce qu'elles sont proligères, proligères en elles-mêmes, par elles-mêmes, par la traduction. C'est de tout cela dont on va parler durant ces quelques jours; c'est de tout cela et encore davantage, la traduction proligère. Proligêrons!

\section{JOYEUX ANNIVERSAIRE À META :}




\section{BIBLIOGRAPHIE}

CHAUNU, Pierre (1989) : Le Grand Déclassement. À propos d'une commémoration, Paris, Robert Laffont. HAGEGE, Claude (1985) : L'Homme de paroles, Paris, Fayard.

HAGËGE, Claude (1987): Le Français et les siècles, Paris, Odile Jacob.

LADMIRAL, Jean-René et Edmond Marc LIPIANSKY (1989): La Communication interculturelle, Paris, Armand Colin. 